\title{
Philosophiques
}

\section{J.-G. Fichte et F.-W.-J. Schelling, Correspondance (1794-1802), présentation, traduction et notes de Myriam Bienenstock, Paris, P.U.F. (coll. "Epiméthée »), 1991, 190 pages.}

\section{Suzanne Foisy}

Volume 20, numéro 1, printemps 1993

URI : https://id.erudit.org/iderudit/027212ar

DOI : https://doi.org/10.7202/027212ar

Aller au sommaire du numéro

Éditeur(s)

Société de philosophie du Québec

ISSN

0316-2923 (imprimé)

1492-1391 (numérique)

Découvrir la revue

Citer ce compte rendu

Foisy, S. (1993). Compte rendu de [J.-G. Fichte et F.-W.-J. Schelling, Correspondance (1794-1802), présentation, traduction et notes de Myriam Bienenstock, Paris, P.U.F. (coll. « Epiméthée »), 1991, 190 pages.] Philosophiques, 20(1), 202-203. https://doi.org/10.7202/027212ar d'utilisation que vous pouvez consulter en ligne.

https://apropos.erudit.org/fr/usagers/politique-dutilisation/ 
J.-G. Fichte et F.-W.-J. Schelling, Correspondance (1794-1802) présentation, traductionetnotes de Myriam Bienenstock, Paris, P.U.F. (coll."Épiméthée"), 1991, 190 pages.

\section{par Suzanne Foisy}

Cette Concspondance révèle une grande partie des enjeux de l'idéalisme transcendantal. Il faut rappeler que la période entre 1794 et 1802 est la période de naissance du romantisme allemand et celle de l'achèvement des différentes versions de la Doctrine de la scicnce de Fichte. L'impact de cette cuvre a su atteindre le jeune Schelling, plus précoce que son compagnon Hegel au Stift de Tübingen. Mais cette époque, c'est aussi celle des inspirations majeures de la philosophie de la nature schellingienne : conception de l'absolu comme Dieu ou nature, idée de force organique s'actualisant dans les Idées pour ume philosophie de la nature (1797). L'Âme dumonde (1798), le Système de lidéalisme transcendantal ( 1800 ) et la Philosophie de l'art ( 1802$)$. Cette traduction ne couvre qu'un temps limité (à l'exception d'une lettre de 94, les textes encerclent les années I799-1802). L'avant-propos d'une quarantaine de pages explique comment cette correspondance prise en elle-mème ne donne pas une image adéquate du vrai débat, bien qu'elle puisse offrir une perspective diffèrente des écrits connus. L'exposition du contexte (historique, universitaire, intellectuel et biographiquel, en portant au jour l'objet occul té à mème l'interprétation conventionnelle des œuvres et des lettres (l'origine et la nature d'une controverse), présente justement ce qui n'est dejà plus contexte et qui préoriente la lecture de ce qui avait semblé révéler au premier abord des tensions personnelles (voir les lettres du I3 septembre et 3 octobre i800, p. 76-87). Les remarques de Myriam Bienenstock font bien surgir le motif de la Différence de Hegel et celui de la fameuse « intuition intellectuelle (dont Schelling reprend l'expression mais à laquelle Fichte avait déjà attribué le contenu). Fichte n'associera jamais Schelling au cercle romantique : première alliance. L'autre accord va se fomenter contre Reinhold. Mais, comme le précise l'auteure de l'avant-propos, était-ce par communauté intellectuelle ou selon les circonstances? Bonne question. Dans l'exposé des problèmes philosophiques contemporains qui cernent selon quelles lignes de combat s'établissent consensus et désaccord, apparaît la fameuse querelle de l'athéisme qui conduira au proces de Fichte et qui fera intervenir Lessing. Mendelssohn, Herder autour de Jacobi (qui avait détecté du fatalisme dans le monisme panthéistique de Spinozal. C'est un procès intenté à la philosophie en général auquel le jeune Schelling va se rallier en déclarant ètre devenu spinoziste dans la conjugaison romantique de la raison et du cceur. La perspective ontologique schellingienne est d'ailleurs plus près de Jacobi que du criticisme. Mais c'est là un danger pour Fichte que Jacobi n'a d'ailleurs jamais accusé d'être athée: c'est plutôt son idéalisme qui est un « spinozisme renversé ». Fichte pour sa part distingue toujours deux points de vue : celui du commun des mortels qui table sur la vie réelle (nature trouvée), celui du philosophe transcendantal qui prétend penser la pensée (nature produite). Il voit dans l'entreprise de Bardili et dans le journal anticritique de Reinhold (i 801 ). une tentative de réhabilitation du dogmatisme précritique. Le séminariste de 
Tübingen s'enthousiasme de ce revirement du maître qui semble reprendre son intuition intellectuelle de l'absolu (lettre du 24 mai I80I, p. IIo). Mais Fichte veut plutôt contrer stratégiquement ses adversaires. Selon Bienenstock, c'est dans ce but seulement qu'il demande à Schelling de ne pas « ébruiter » leurs différences (3i mai ı8or, p. I14-123). Un autre désaccord fondamental réside dans la façon de concevoir les relations entre la philosophie transcendantale et la philosophie de la nature (i5 novembre i800, p. 94-97). Schelling semble avoir cru naïvement que Fichte se serait rallié à sa position ( 15 nov-27 déc. 1800 ). Jusqu'en mai I8or, il veut éperdument situer son propre système au fondement de l'unité de leurs vues, tout en croyant avoir été fidèle à une intention fichtéemne dans la construction de sa Philosophie de la nature (inspirée des questions de la troisième Critique kantienne). Cette philosophie répond au problème que l'histoire de la philosophie avait laissé ouvert: comment se fait-il que les phénomènes naturels observent une suite continue? C'est que la nature « exprime » nécessairement les lois de notre esprit (elle est l'« esprit visible », et l'esprit la « nature invisible »). Or, ce projet diffère de celui de Fichte, plus fidèle au transcendantalisme kantien. Schelling n'arrivera malheureusement pas à clarifier le chemin bidirectionnel de la nature au moi et Fichte y détectera un cercle ( 5 nov. I800, p. 95, 3I mai I80I, p. II4-123). Pour Mme Bienenstock, élaborer une philosophie de la religion aurait atténué les autres différends. Mais la foi de Fichte n'était pas celle de Jacobi.

Une « Note sur la traduction » (p. 47-48) nous informe sur le caractère scientifique et philologique des diverses éditions de cette Correspondanceet nous fait part des choix de style de la traductrice. Le tout se poursuit avec trois appendices inédits: Schelling, « Déduction générale du processus dynamique », $\S 63$; Fichte, « A la lecture de l'idéalisme transcendantal de Schelling » et «Propositions visant à élucider l'essence des animaux 》. Après cet ensemble de textes, on lira les notes de la traduction (p. $155^{-176)}$ ), les indications bibliographiques assez complètes en allemand et en français (textes des auteurs, textes contemporains de leur époque, études sur les auteurs, études sur la philosophie de l'époque et le débat) (p. 177-183) et l'index nominum qui clôt le volume.

Au-delà de l'anecdote qui éclairerait de son flambeau une partie seulement des œuvres en question, on peut décrypter l'admiration de Schelling envers Fichte dans cet « entièrement vôtre » si longuement répété au bas de chaque lettre ainsi que le détachement affectif progressif dans les signatures et les adieux au fil de la controverse qui s'accentue jusqu'aux irrémédiables déclarations d'octobre 1801 et de janvier 1802 . Si la lecture de la Correspondance nous dévoile qu'il n'est pas adéquat de rapporter simplement le débat au problème des relations du transcendantal et du naturel, l'avant-propos nous révèle dans le même sens que le désaccord sur ces relations est inconcevable sans un approfondissement de la querelle de l'athéisme auquel il nous convie. Une chose est sûre : les lettres et leur commentaire consacrent la paternité et la crédibilité de Fichte et nous incitent à connaître Schelling ailleurs, justement dans ses écrits pour la plupart récemment traduits dans la même collection. 